Volume 9, No.4, July - August 2020

International Journal of Advanced Trends in Computer Science and Engineering

Available Online at http://www.warse.org/IJATCSE/static/pdf/file/ijatcse68942020.pdf

https://doi.org/10.30534/ijatcse/2020/68942020

\title{
Safe Route Recommendation System for Pedestrians
}

\author{
Anurag $\mathbf{P}^{1}$, Kshitij $\mathrm{K}^{2}$, Srividhya $\mathbf{S}^{*^{3}}$ \\ ${ }^{1}$ Department of Information and Technology, SRM Institute of Science and Technology, Chennai, India, \\ anuragpatidar17@gmail.com. \\ ${ }^{2}$ Department of Information and Technology, SRM Institute of Science and Technology, Chennai, India, \\ kshitijkhune@gmail.com. \\ 3*Assistant Professor, Department of Information Technology, SRM Institute of Science and Technology, \\ Chennai, India, srividhs1@ srmist.edu.in
}

\begin{abstract}
To propose a System that transits a safe route to the user from the source to the destination by taking into account the vulnerabilities between the two and thereby act accordingly upon them and propose the safest possible route to the user. For decisive route navigation, we need to assess the crime rate in real time and there by reroute the user to the destination if necessary. Our reckoned system also produces a heat-map, geo-scope and a scatter-plot by analysing and depicting the crime-rate dynamically. The system also allows the user to share the real-time location in-case if they feel a particular area is unsafe whose co-ordinates are dynamically updated. In order to verify the updated data from various sources, a police verification portal is provided which verifies the data from the local police station through a secured gateway. The safe route recommendation system aims at reducing the crime rate with technical superiority and thereby bringing a sense of comfort on the way we travel.
\end{abstract}

Key words : Safe route, Google Maps, Crime-rate, Hot-spot, Recommendation

\section{INTRODUCTION}

The impact of the increased crime rate has drastically affected the mental health of people and created a sense of threat in the minds of the people. A recent survey proclaimed that the problem of large cities in developing countries expressed a high scale rate of crime and issue of enormous urban communities in creating nations communicated in high sizes of wrongdoing and a few different emergencies. Besides, huge urban areas are comprehensively known as spots of more wrong doing and individual risk. Factually, the quantity of theft and merchandise violations shows the keenest ascent with urban scale. Along these lines, it is important to think about another angle, which is the security viewpoint. One of the primary reasons of the expansion in wrong doing of burglary or theft in transit is the absence of investigation of security issues in the primary stage. At characterized hazard into three principle segments: (a) the event of an occasion (communicated as a likelihood or recurrence of certain bothersome occasions happened); (b) defencelessness as a proportion of the weakness of the items to be shielded from unfortunate occasions; (c) presentation speaks to the weighted estimation of individuals, products and foundation influenced during and after the occasion.

A few examinations [6], [7] have been done to tackle this issue. The sheltered way here intends to the streets that are liberated from wrong doing history. This exploration chooses to consider at the requirement for an application to give the most secure street proposals by the travellers while travelling from one place to another. We propose an online application utilizing Google Maps API that can be gotten to by individuals to consider their safety in a particular region by allowing sharing their real time location. The system considers various explicit factors that may act as the vulnerabilities and tackle them in order to provide a safe way to the travellers. The analysis of the explicit factors is done by using the feedback provided to the locals around that particular area which in turn have to be verified by the local police station in order to avoid the wrong doing.

Several works like in [9], [15] were related to identification of safest route for the travel. The paper has been framed as follows. Section 2 is related to the survey on the articles related to safe route recommendation using different ways. Next section 3 concentrates on problem statement. In section 4 the detailed architecture has been discussed. Section 5 deals with the modules of the work and section 6 with results and discussions. Finally it is concluded along with future work.

\section{LITERATURE SURVEY}

In [1], A. Jain et al, emphasises on creating a geo-system that will create a safe passage of route for women by calculating the crime rate of that particular route. It calculates a danger index which helps in reckoning the route by assessing the crime rate. As per the results the system is solely dependent on asynchronous data which we overcome in our model by developing a real-time system that allows user to share the location and dynamically reflect it in the system. 
Anurag P et al., International Journal of Advanced Trends in Computer Science and Engineering, 9(4), July - August 2020, 4665 - 4670

E. Dumbaugh et al, explains the existing facts focusing on the lead crashes of child pedestrians and thereby develop a program for schools that focuses on building a system that recommends safe route to the children by taking into account the ten safety counter-measures [2]. It determines the user behaviour that results in such accidents. The study indicates that the system is build by considering the human-behavioural model which can sometimes lack precision. Our proposed model also focuses on the external vulnerabilities that can lead to the crashes and reckon a safe passage of route.

A. Utamima et al, in [3] describes the fact that the Google map shows the shortest distance route and doesn't cover the safety level. They suggested an application which will recommend for the safest route for users from source to destination. It has shown the cast study for Surabaya dataset, a city in Indonesia.

Machine-learning algorithms are used to generate the risk score of a particular route based upon average risk score of nearby clusters/regions. S. Soni et al, in [4] taken the crime dataset from NYC Open Data to determine the average risk score of clusters/regions. They suggest that better results can be achieved by increasing the factors of safety level. The aim to make a better prediction algorithm that can identify crimes while travelling on a particular route.

A hybrid approach for finding safe routes using semantic processing and classification Bayes algorithms was proposed in [5] by F. Mata et al. They found the optimal path by not only considering the shortest distance but also keep mark on other factors such as security level. Authors considered the need for an application to recommend the safest road to be passed by the users while crossing through a particular area. In [10] they examines about a set of methodologies which when applied to the traffic signal, will help in defeating the issue of traffic blockage related issues. It works in the accompanying three angles: Development of an assortment of street crossing, and proposes complex changes of the street crossing traffic light frameworks. The control arrangement that isn't just ready to maintain a strategic distance from the traffic clog scattering yet additionally to stay away from new traffic blockages being occurred. It assesses the adequacy of our control procedures for taking care of the issue of traffic clog in three sorts of traffic stream circumstances by utilizing VISSIM.

In [8], talked about a top to bottom execution of savvy road light framework for shrewd city with electronic administration framework. Specifically, the proposed design contains road lights in gatherings, which associated with online administration framework to give interface to approved client. They characterized a calculation to find solicitation to answer transmission between the used system and the other way around alongside changing between the set shading temperatures alongside also darkening usefulness if essential. Thus roadlight is then constrained by a focal server known as road light server in which control flagging experience is done by organizer initially at that point every switch as per their ID.

$\mathrm{Bi}, \mathrm{C}$ et al. in [11] proposed a method using auto encoder and markov decision model for predicting the optimized global route for the safe evacuation.

Galbrun, E et al. developed an application for safe path identification in the streets of urban city of Chicago. They identified the path using two objectives based on path and also risk of the path in [12].

In [13], Levy, S et al. suggested an optimized path using reinforcement technique that enables for prediction of safe routes. Rewards based algorithm is implemented using deep learning techniques to choose the best route that hold for high reward. The model was trained for the Boston city as hotspot region.

Siya Bao et al. in [14] implied a path for finding the route for the pedestrians using the parameters street lightning, landmark of the place in terms of visibility and scarcity, turning in the street, etc. Authors did real time experiments with the pedestrians and gives them notification regarding the finest route for their destination place.

Problem Statement

Authors in [16] discussed about route generation for travel using candidate data. The increased crime rate has created high sense of wrong doing in the society. Crime data recommendation is done [17]. In order to prevent the mishap, we propose a system which recommends a safe route to people between two endpoints by analysing the vulnerabilities and assessing the crime rate among routes in order to provide the safest route possible. The threat of the crime has been scaled on a crime scale which determines the severity of the crime. The system also tracks the user's movement which enables the user get a vision whether they are moving towards the hotspot zone.

\section{PROPOSED ARCHITECTURE}

Initially, from the crime dataset, latitude and longitude of the crime locations are identified and marked it with a red flag on the Google maps. When users will search for a particular route, the system will show them the route with the unsafe points on the route or near the route with a red flag if exists.

A portal has created and street profile on the basis of user's information is build there. So, whoever will be using the portal for getting the safest route for their journey, has to fill the audit page after completing their journey of that particular route. The Audit page would consist of certain questions, asking them about the experience or basically how they felt 
Anurag P et al., International Journal of Advanced Trends in Computer Science and Engineering, 9(4), July - August 2020, 4665 - 4670

after crossing the street. In figure 1 , the system will ask the user to access their location, to fetch the name of the location with latitude and longitude, and other information like the number of the street light on the route, condition of roads with the same options, number of people on the street, number of the police station on the street, the frequency of transport, all these questions with radio button options like high, medium, less. Then other questions like Major crime incidents on this route with options like rape, murder, kidnapping, loot, tease the duration of crime incidents, like day or night. Users have to compulsory fill all this information or else they won't be able to access the portal next time.

After collecting all this information into the database, the portal will generate a street profile with the majority of votes as an answer to each question similar to Figure 2. For example, asking for a particular route about the number of the street light, out of 100 users, 60 users selected "High" option, 20 users selected "Medium" option, and 10 users selected "less" option. So, the system will select the majority of votes as an answer to that particular question. The system will do the same for all other questions and would generate a street profile. An example of street profile is given in the Figure 3.

This street profile will be sent to police verification, every week automatically through the script. There will be a separate portal for police verification. The system will fetch the street profile from the database and will be sent to the database of police portal. The police in charge will have access to the police portal and can verify the answers of all the questions in the street profile, from their past records like FIR, online complaints. As soon as the police in charge verify the details of the street profile, the details will be fixed. And then an automated mail will be sent to all the localities, mentioning about the new street profile for that particular area.

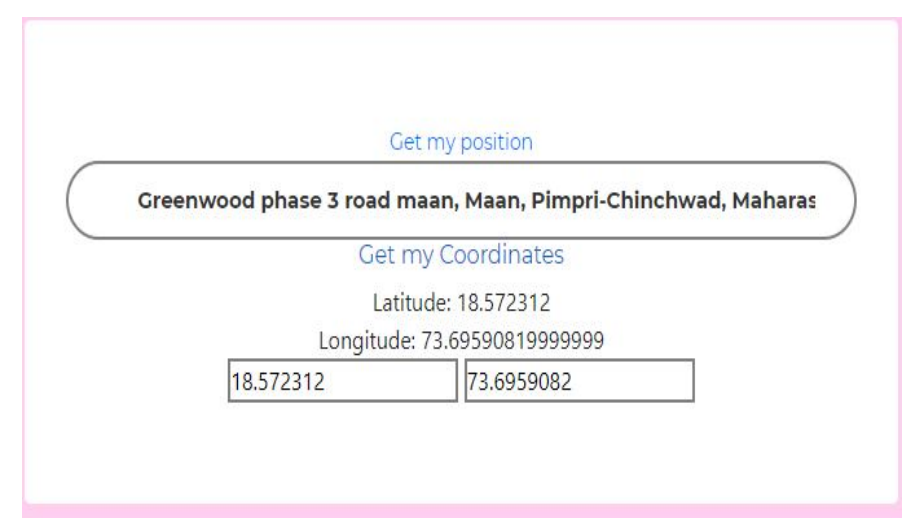

Figure 1: Identify the coordinates of location from Google Map

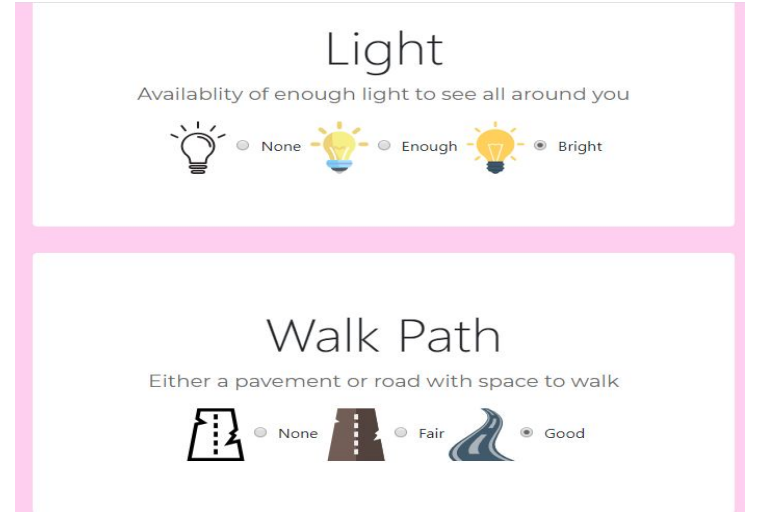

Figure 2: Options for giving user data related to street

\begin{tabular}{|l|l|}
\hline Street profile in Database \\
\hline Street Name & ABC Road \\
\hline Latitude & 71.298 \\
\hline Longitude & 82.268 \\
\hline Number of Street light & Less \\
\hline Condition of road & Good \\
\hline Number of people & Less \\
\hline Number of Police Station & Less \\
\hline Number of Transport & Medium \\
\hline Major Crime & Kidnapping \\
\hline Time for Major Crime & Night \\
\hline How most people felt there & Unsafe \\
\hline
\end{tabular}

Figure 3: Street Profile Example

In the third part, the street profile data and the dataset will be combined into a single table in the database. So, whenever users would type the source and destination location in the system, the system will show the possible routes with the unsafe points (red flag) along with the details of the street profile if exists. And if there are more than one unsafe points on the street, users can compare the details of those unsafe points through their street profile.

In the fourth part, we are using web wrapping to extract the necessary information from the articles posted by users on the website to drawn an enclosed area with red colour claiming as some protest or riots are going on that area. The web scraping module will search for some particular keywords like protests/ riots, with time and date, then matching with the current time and date, just to confirm that the information is not outdated. If its matches with the current time, date it will fetch other necessary information like location, details regarding protest or riot. Then this information will be verified by the police portal, if the information is true, means accepted by the police, it will draw a red area signalling a danger zone on the map. If suppose no one turns up for posting the article, police can also mark the whole area as a danger zone on their sources of information, and the system will act accordingly.

In the fifth part, will are analysing the data for the future purpose to make the portal work more accurate, for analysing 
the crime incidents and the curve, we are generating a heatmap through google cloud API. Heatmap describes the magnitude or density of the crime incident on the map, that how the crimes are clustered and their pattern and along with this we are generating a clustered map using machine learning libraries, the map is generated every month, the screenshot of the map is taken every end of the month to compare the distribution of crime spots and frequency, with the next month.

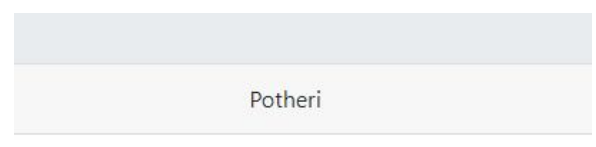

\subsection{5}

\subsection{5}

\section{Accept Reject}

Figure 4: Accepting ration of the route

\section{ARCHITECTURE DIAGRAM}

Modules in the proposed architecture as shown in Figure 5 are described below.

\section{A. Web Scrapping:}

Web scrapping means extracting the raw data from the available sources. The web scraped data is used in Hotspot module to generate an enclosed area which would be depicting as a danger zone. The users can paste the links of the articles on the website and then the module will extract the necessary information like incident, time, date, day, location, longitude, longitude and will send all these information to the database. The hotspot module will fetch the data from the database collected by web scrapping module and will generate an enclosed area on the basis of information.

\section{B. Audit Page :}

This module consists of an HTML form asking a certain number of questions like the number of street lights, number of people, nearest police station, crime scenes, how the user felt after crossing that street, all these questions for a particular route to users. Any of the users after crossing the street can fill these information in the form.

\section{Police Portal :}

There is a separate police portal, where police in charge can crosscheck the information obtained in the database of the system with the number of FIR's for that particular area and can accept or reject the verification accordingly. As soon as they accept the verification for a particular area, an automated mail will be sent to all the localities that a new unsafe point has been added in your area, check the information online and be aware.

\section{Google Map Route :}

This module consists of a google map, the users can put their source and destination location in the input field, then the system will show the route with unsafe points on google map if exists. If there are more than one unsafe point, users can compare them on the basis of crime rate, nearest police station, number of crowds, number of street lights etc.

\section{E. Hotspot Map :}

This module basically tells you about the area where riots or protests are going on. It tells the users that the whole area is under danger zone which is depicted by red colour in the map.

F. Crime Analysis using HeatMap :

This module consists of a heatmap. This map is used for the analysis purpose. It shows the magnitude of crimes on a particular route with two colours. It gives a visual image of how the crimes are clustered. Heatmap is generated using the dataset download from Kaggle.

G. Crime Analysis using Machine Learning :

A python script is used to integrate the data to the map of the city using latitude and longitude as the parameters. A scatter-plot like structure is being plotted on the map of the city where each point depicts the latitude and longitude of the region of crime occurrence which can be later used to analyse and predict which area has the most number of crimes and thereby take the safety precautions accordingly to reduce the rate of crime. Pandas, matplotlib, plotly are the packages that are being used to avail the necessary results.

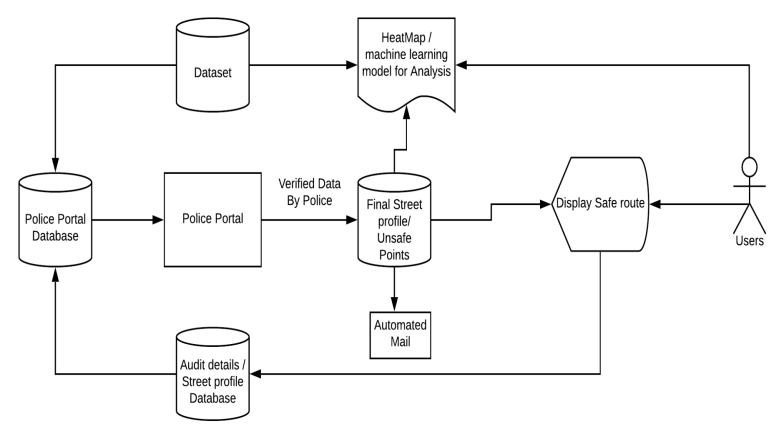

Figure 5: Architecture Diagram

\section{SIMULATION RESULTS}

\section{A. Google Maps Route}

The route between source A and destination B with 2 unsafe points are depicted by red flags in the Figure 6 and Figure 7 depicts the crime scene rate. The users can see more precise information by tapping the red flag, and can compare if there exists more than one points. 




Figure 6: Depiction of unsafe path



Figure 7: Crime rate notification

\section{B. Hotspot Map}

The whole red area describes that some protest or riots are going on in that area as shown in Figure 8.

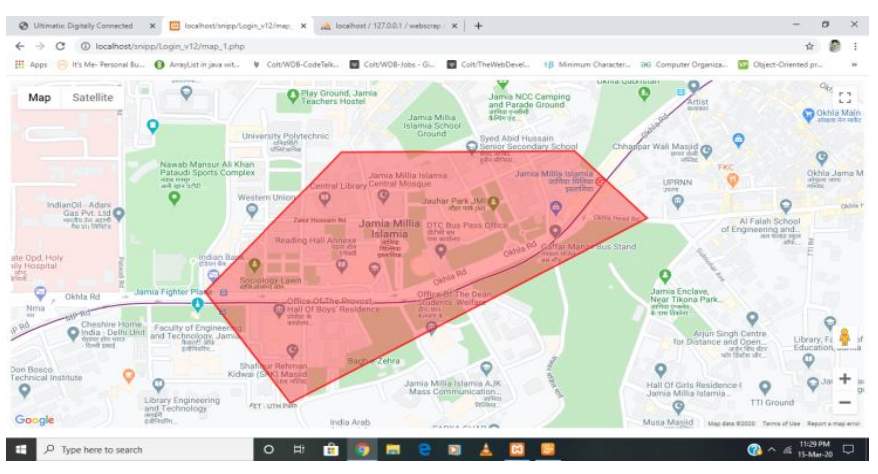

Figure 8: Hot Spot area

\section{Crime Analysis using HeatMap}

Figure 9 shows the magnitude of crimes on a particular route with two colours. It gives a visual image of how the crimes are clustered.

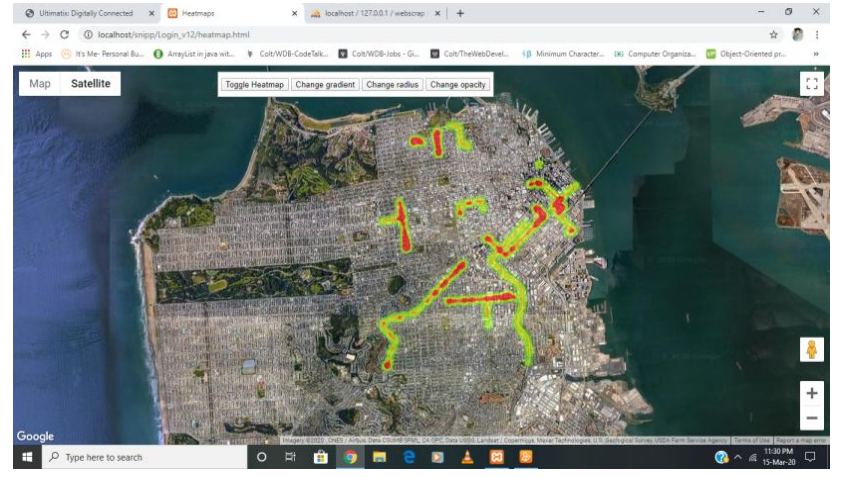

Figure 9: Visualization of Crime regions

\section{CONCLUSION AND FUTURE WORK}

In the proposed architecture, a navigation model that successfully recommends a safe route to the users by assessing the vulnerabilities between multiple destinations has been built. The data collected from the different sources is filtered here and information is verified through a police-verification portal and further integrated into the prototype. By analysing the vulnerabilities and the user's current location the system rates the routes to the destination on a crime -scale, thereby recommending the safest possible route to the user. The results achieved by the system using various The results achieved not only ensure the technical superiority but also the user-friendly prospect of the methodology. A part of information to build the model is achieved by studying the rational behaviour of numerous individuals. In the near future, we have planned to build and train a machine learning model that can be integrated with this system to predict the area of crime beforehand and thus prevent it from happening. The accuracy of the model would be tested before integrating it into the system in order to avoid any mishap. The future work also anticipates to increase the geographical outbound of the API which is not strong towards the rural areas.

\section{REFERENCES}

1. Chakraborty, Goutam, Dynamic distribute route recommendation system for multiple destinations, 12th IEEE International Conference on Electrical Engineering/Electronics, Computer, Telecommunications and Information Technology (ECTI-CON), pp. 1-5, 2015.

2. Jain, S. Sharma, H. Kumar, D. Parashar, Geographical information system based safe path recommender, Int. J. Innov. Technol. Explor. Eng., vol. 8, no. 10, pp. 3863-3868, 2019. https://doi.org/10.35940/ijitee.J9895.0881019

3. Dumbaugh, L. Frank, Traffic safety and safe routes to schools: Synthesizing the empirical evidence, Transp. Res. Rec., pp. 89-97, 2007.

4. Utamima, A. Djunaidy, Be-safe travel, a web-based geographic application to explore safe-route in an area, AIP Conf. Proc., vol. 1867, 2017. 
5. S. Soni, V. G. Shankar, and S. Chaurasia, Route-the safe: A robust model for safest route prediction using crime and accidental data, International Journal of Advanced Science and Technology, vol. 28, no. 16, pp. 1415-1428, 2019.

6. Mata, A Mobile Information System Based on Crowd-Sensed and Official Crime Data for Finding Safe Routes: A Case Study of Mexico City, Mob. Inf. Syst., 2016. https://doi.org/10.1155/2016/8068209

7. M. U. S. Khan et al., MacroServ: A Route Recommendation Service for Large-Scale Evacuations, IEEE Trans. Serv. Comput., vol. 10, no. 4, pp. 589-602, 2017.

8. E. Zhou, S. Mao, and M. Li, Investigating street accident characteristics and optimal safe route recommendation: A case study of New York City, Int. Conf. Geoinformatics, 2017.

9. M. Lujak and S. Ossowski, On avoiding panic by pedestrian route recommendation in smart spaces, IEEE Int. Black Sea Conf. Commun. Networking, BlackSeaCom 2016.

10. R. Verma, S. Ghosh, M. Saketh, N. Ganguly, B. Mitra, S. Chakraborty, ComFride: A smartphone based system for comfortable public transport recommendation, 12th ACM Conf. Recomm. System, pp. 181-189, 2018. https://doi.org/10.1145/3240323.3240359

11. Bi, C., Pan, G., Yang, L., Lin, C.C., Hou, M., Huang, Y., Evacuation route recommendation using auto-encoder and Markov decision process, Applied Soft Computing, 2019.

12. Galbrun, E., Pelechrinis, K., Terzi, E., Urban navigation beyond shortest route: The case of safe paths, Information Systems, pp.160-171, 2017.

13. Levy, S., Xiong, W., Belding, E., Wang, W.Y., SafeRoute: Learning to Navigate Streets Safely in an Urban Environment, arXiv preprint arXiv:1811.01147.

14. S. Bao, T. Nitta, K. Ishikawa, M. Yanagisawa, N. Togawa, A safe and comprehensive route finding method for pedestrian based on lighting and landmark, 5th IEEE Global Conference on Consumer Electronics, Kyoto, pp. 1-5, 2015. https://doi.org/10.1109/GCCE.2016.7800525

15. K. H. Yew, T. T. Ha, S. D. S. J. Paua, SafeJourney: A pedestrian map using safety annotation for route determination, International Symposium on Information Technology, Kuala Lumpur, pp. 1376-1381, 2010.

16. T, Jisna. Efficient Route Recommendation System Based On Keyword Using Candidate Route Generation And Travel Route Exploration, International Journal of Advanced Trends in Computer Science and Engineering.vol. 8,pp. 419-425, 2019. https://doi.org/10.30534/ijatcse/2019/15832019

17. Chua, Everly. Crime Data Forecasting using Exponential Smoothing, International Journal of
Advanced Trends in Computer Science and Engineering, vol. 9,pp. 69-75, 2020.

https://doi.org/10.30534/ijatcse/2020/1391.12020 